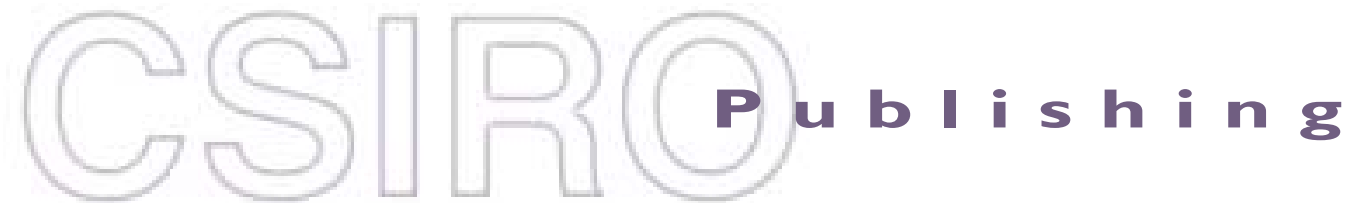

\section{Publications of the Astronomical Society of Australia}

Volume 19, 2002

(C) Astronomical Society of Australia 2002

An international journal of astronomy and astrophysics

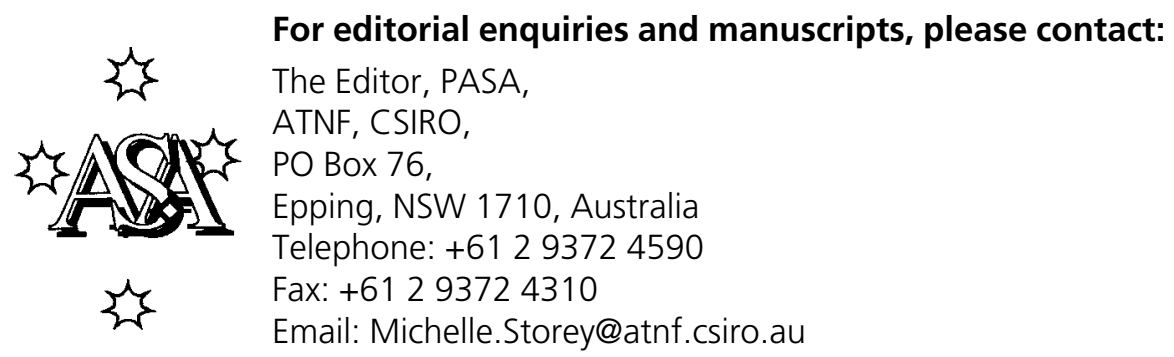

For general enquiries and subscriptions, please contact: CSIRO Publishing PO Box 1139 (150 Oxford St)

Collingwood, Vic. 3066, Australia

Telephone: +6139662 7666

Fax: +61 396627555

Email: publishing.pasa@csiro.au

C S I RO

PUBLISHING Published by CSIRO Publishing

for the Astronomical Society of Australia

www.publish.csiro.au/journals/pasa 


\title{
Effects of Lower Boundary Conditions on the Stability of Radiative Shocks
}

\author{
Curtis J. Saxton \\ Department of Physics and Theoretical Physics, Faculty of Science, and \\ Research School of Astronomy and Astrophysics, \\ Australian National University, Canberra, ACT 0200 \\ saxton@mso.anu.edu.au \\ Received 2001 October 19, accepted 2002 March 11
}

\begin{abstract}
Thermal instabilities can cause a radiative shock to oscillate, thereby modulating the emission from the post-shock region. The mode frequencies are approximately quantised in analogy to those of a vibrating pipe. The stability properties depend on the cooling processes, the electron-ion energy exchange, and the boundary conditions. This paper considers the effects of the lower boundary condition on the postshock flow, both ideally and for some specific physical models. Specific cases include constant perturbed velocity, pressure, density, flow rate, or temperature at the lower boundary, and the situation with nonzero stationary flow velocity at the lower boundary. It is found that for cases with zero terminal stationary velocity, the stability properties are insensitive to the perturbed hydrodynamic variables at the lower boundary. The luminosity responses are generally dependent on the lower boundary condition.
\end{abstract}

Keywords: accretion — shock waves — binaries: close — white dwarfs

\section{Introduction}

A system that depends on the length- or time-scales of radiative cooling or other energy exchange processes may be thermally unstable (Field 1965) if perturbations from the initial or equilibrium condition alter the cooling scale in a manner that enhances the perturbation. The effects of radiative cooling are expressed in terms of a function of local hydrodynamic variables such as density and temperature. Qualitatively, a thermally unstable system has a form of cooling function which increases the cooling length when the shock temperature increases and conversely the cooling length decreases when the shock temperature lowers. Langer, Chanmugam, \& Shaviv (1981) discovered such a thermal instability in the radiative accretion shocks of white dwarfs (see e.g. reviews by Cropper 1990; Wu 2000). This instability was verified by subsequent studies using different numerical techniques (e.g. Imamura, Wolff, \& Durisen 1984), and linear stability analyses (e.g. Chevalier \& Imamura 1982).

Linear analyses (Chevalier \& Imamura 1982; Imamura et al. 1996; Saxton et al. 1998; Saxton \& Wu 1999) indicate that the shock oscillations have a sequence of eigenmodes. If bremsstrahlung dominates the cooling then there tends to be a fundamental mode stable against oscillations, and unstable overtones. Depending on its relative efficiency, cyclotron cooling tends to stabilise modes, except when the electron-ion energy exchange is comparatively inefficient, in which case modes can be destabilised with increasing cyclotron efficiency.

Tóth \& Draine (1993) found that when bremsstrahlung cooling is the dominant cooling process the frequencies resemble those of a pipe open at one end (oscillation frequency $f \propto n-\frac{1}{2}$ for integer harmonic number $n$ ).
This pattern persists for two-temperature shocks when the electron-ion exchange is efficient (Imamura et al. 1996; Saxton \& Wu 1999; Saxton 1999). When cyclotron cooling is very efficient, so that the electron-ion exchange process is unable to maintain strong thermal coupling between electrons and ions, the modes are more like those of a doubly-closed or doubly-open pipe, $f \propto n$ (Saxton $\& \mathrm{Wu}$ 1999).

The standard analytic treatment defines the lower boundary as the place where the flow velocity becomes zero in the time-independent solution and does not oscillate in the time-dependent response to perturbation (e.g. accreted material diffuses across a stellar surface very slowly compared to the oscillatory timescale). The pressure, density, and other hydrodynamic variables are nonzero and are allowed to oscillate. Different numerical investigations treated the lower boundary differently: e.g. dense matter near the lower boundary was deleted in Imamura (1985), was merged with neighbouring cells in Imamura et al. (1984), and may have had its gradients softened by the spatial filter on the Eulerian grid of Langer et al. (1981). These diverse treatments produced some different results. Langer et al. $(1981,1982)$ found a fundamental mode that is unstable to oscillations, whereas other studies (e.g. Imamura 1985; Imamura et al. 1984; Wolff, Wood, \& Imamura 1991; Wood, Imamura, \& Wolff 1992) found that the first unstable mode is the first overtone, and the fundamental mode is damped, consistent with stability analyses. The collaborations also produce different results regarding the phasing of oscillatory modulations of the bremsstrahlung and cyclotron luminosities.

This paper investigates the role of the lower boundary in relation to the stability and emission properties of 
post-shock flows. This issue has not been thoroughly discussed in previous studies of one-temperature (Saxton et al. 1998) and two-temperature (Saxton \& Wu 1999, 2001) radiative shocks. In an appendix, Imamura et al. (1996) stated that changing the lower boundary condition from constant velocity to constant pressure did not affect their results with a power-law cooling function. The present work explores different analytic choices of the lower boundary condition, in the presence of two cooling processes and inequality between the electron and ion temperatures. White dwarf accretion shocks are an illustrative case study. The conditions considered herein include constant pressure, density, flow rate, and temperature at the white dwarf surface, or a boundary with nonzero longitudinal velocity (e.g. due to lateral 'leakage' transverse to the magnetic field lines).

The plan of the paper is as follows. Section 2 outlines the general formulation, and Section 3 describes the cases of different boundary conditions investigated. Section 4 presents and discusses the local and global stability properties. Section 5 examines how the different lower boundary conditions affect the modulation of the luminosities of the oscillating post-shock flow. Section 6 draws the conclusions.

\section{Perturbative Analysis}

The hydrodynamics of the accretion flow are governed by the equations for continuity of mass, momentum, total energy, and electron energy:

$$
\begin{gathered}
\left(\frac{\partial}{\partial t}+\mathbf{v} \cdot \nabla\right) \rho+\rho(\nabla \cdot \mathbf{v})=0 \\
\rho\left(\frac{\partial}{\partial t}+\mathbf{v} \cdot \nabla\right) \mathbf{v}=-\nabla P \\
\left(\frac{\partial}{\partial t}+\mathbf{v} \cdot \nabla\right) P-\gamma \frac{P}{\rho}\left(\frac{\partial}{\partial t}+\mathbf{v} \cdot \nabla\right) \rho=-(\gamma-1) \Lambda
\end{gathered}
$$

and

$$
\begin{aligned}
& \left(\frac{\partial}{\partial t}+\mathbf{v} \cdot \nabla\right) P_{\mathrm{e}}-\gamma \frac{P_{\mathrm{e}}}{\rho}\left(\frac{\partial}{\partial t}+\mathbf{v} \cdot \nabla\right) \rho \\
& \quad=(\gamma-1)(\Gamma-\Lambda)
\end{aligned}
$$

where $\mathbf{v}, \rho, P$, and $P_{\mathrm{e}}$ are the fluid velocity, density, total pressure, and electron pressure, and $\gamma$ is the adiabatic index (assumed equal to $5 / 3$ for an ideal monatomic gas). The radiative loss term $\Lambda=\Lambda_{\text {br }}+\Lambda_{\text {cy }}$ comprises a sum of energy losses due to bremsstrahlung and cyclotron cooling. The functional form of $\Lambda$ was described in $\mathrm{Wu}$ (1994):

$$
\Lambda \equiv \Lambda_{\mathrm{br}}+\Lambda_{2}=\Lambda_{\mathrm{br}}\left[1+\epsilon_{\mathrm{s}}\left(\frac{P_{\mathrm{e}}}{P_{\mathrm{e}, \mathrm{s}}}\right)^{\alpha}\left(\frac{\rho}{\rho_{\mathrm{s}}}\right)^{-\beta}\right]
$$

where $\rho_{\mathrm{s}}$ and $P_{\mathrm{e}, \mathrm{s}}$ are the density and electron partial pressure at the shock, and the bremsstrahlung cooling function is $\Lambda_{\text {br }}=A \rho^{2}\left(P_{\mathrm{e}} / \rho\right)^{1 / 2}$ with $A \approx 3.9 \times 10^{16}$ in cgs units (see e.g. Rybicki \& Lightman 1979). The parameter $\epsilon_{\mathrm{s}}$ is the efficiency of the second cooling process compared to bremsstrahlung cooling, evaluated at the shock. For the case of cyclotron-emitting post-shock regions of accreting white dwarfs, the indices are $\alpha=2.0, \beta=3.85$.

The term $\Gamma$ is the electron-ion energy exchange (e.g. Spitzer 1962; Melrose 1986; Imamura et al. 1996),

$$
\Gamma=\frac{4 \sqrt{2 \pi} e^{4} n_{\mathrm{e}} n_{\mathrm{i}} \ln C}{m_{\mathrm{e}} c}\left[\frac{\theta_{\mathrm{i}}-\left(m_{\mathrm{e}} / m_{\mathrm{i}}\right) \theta_{\mathrm{e}}}{\left(\theta_{\mathrm{e}}+\theta_{\mathrm{i}}\right)^{3 / 2}}\right],
$$

where $m_{\mathrm{i}, \mathrm{e}}, T_{\mathrm{i}, \mathrm{e}}, \theta_{\mathrm{i}, \mathrm{e}}=k_{\mathrm{B}} T_{\mathrm{i}, \mathrm{e}} / m_{\mathrm{i}, \mathrm{e}} c^{2}$, and $n_{\mathrm{i}, \mathrm{e}}$ are the ion and electron masses, temperatures, dimensionless temperatures and number densities of ions and electrons respectively. The other constants are $k_{\mathrm{B}}$ the Boltzmann constant, $c$ the speed of light, $e$ the electron charge, and $\ln C$ is the Coulomb logarithm.

Normalising the hydrodynamic quantities by their respective pre-shock values $\left(v_{\mathrm{ff}}\right.$ the free-fall velocity, $\rho_{\mathrm{a}}$ the pre-shock density of the flow, and $x_{\mathrm{s} 0}$ the equilibrium shock height), the variables of the stationary solution are defined as $\tau_{0}=v / v_{\mathrm{ff}}, \pi_{0}=P / \rho_{\mathrm{a}} v_{\mathrm{ff}}^{2}$, and $\pi_{\mathrm{e}}=P_{\mathrm{e}} / \rho_{\mathrm{a}} v_{\mathrm{ff}}^{2}$ for the flow velocity, total pressure, and electron pressure. The hydrodynamic variables are functions of the vertical position coordinate $\xi \equiv x / x_{\mathrm{s}}$, where $x_{\mathrm{s}}$ is the instantaneous shock height, as distinct from the equilibrium value. Then the cooling function and electron-ion exchange function are

$$
\begin{aligned}
& \Lambda=\left(\rho_{\mathrm{a}} v_{\mathrm{ff}}^{3} / x_{\mathrm{s} 0}\right) \psi_{\mathrm{c}} \tau_{0}^{3 / 2} \pi_{\mathrm{e}}^{1 / 2}\left[1+\epsilon_{\mathrm{s}} f\left(\rho_{\mathrm{a}}, P_{\mathrm{e}}\right)\right] \\
& \Gamma=\left(\rho_{\mathrm{a}} v_{\mathrm{ff}}^{3} / x_{\mathrm{s} 0}\right) \psi_{\mathrm{c}} \psi_{\mathrm{ei}} \tau_{0}^{-5 / 2} \pi_{\mathrm{e}}^{-3 / 2}\left(1-\tau_{0}-2 \pi_{\mathrm{e}}\right)
\end{aligned}
$$

where the constant $\psi_{\mathrm{c}}=A x_{\mathrm{s} 0} \rho_{\mathrm{a}} / v_{\mathrm{ff}}$ and $\psi_{\text {ei }}$ parameterises the efficiency of electron-ion energy exchange compared to the radiative cooling. The conditions at the shock $(\xi=1)$ are $\tau_{0}=\frac{1}{4}, \pi_{0}=\frac{3}{4}, \pi_{\mathrm{e}}=\frac{3}{8}\left(1+\sigma_{\mathrm{s}}^{-1}\right)^{-1}$, where $\sigma_{\mathrm{s}} \equiv\left(P_{\mathrm{e}} / P_{\mathrm{i}}\right)_{\mathrm{s}}$ is the electron to ion pressure ratio at the shock. The stationary state of the system is completely specified by the parameters $\left(\sigma_{\mathrm{s}}, \psi_{\mathrm{ei}}, \epsilon_{\mathrm{s}}\right)$.

As in Imamura et al. (1996) and Saxton \& Wu (1999), the shock position is perturbed about its equilibrium position, $x_{\mathrm{s}}=x_{\mathrm{s} 0}+x_{\mathrm{s} 1} e^{\omega t}$, with complex frequency $\omega \equiv \delta v_{\mathrm{ff}} / x_{\mathrm{s} 0}$. The stationary solution is obtained by separating the zeroth order terms in the hydrodynamic equations, involving the total pressure $\pi_{0}$ and electron pressure $\pi_{\mathrm{e}}$.

The perturbation of the post-shock structure is described by a set of differential equations, with the complex variables $\lambda_{\zeta}(\xi), \lambda_{\tau}(\xi), \lambda_{\pi}(\xi)$, and $\lambda_{\mathrm{e}}(\xi)$ corresponding to perturbations of density, longitudinal velocity, total pressure, and electron pressure respectively. The $\lambda$ functions are normalised by $\varepsilon \equiv x_{\mathrm{s} 1} \omega / v_{\mathrm{ff}}$, the relative amplitude of the oscillation of the shock position. The instantaneous density, velocity, total pressure, and electron pressure are

$$
\begin{aligned}
& \rho(\xi, y, t)=\rho_{\mathrm{a}} \cdot \zeta_{0}(\xi)\left(1+\varepsilon \lambda_{\zeta}(\xi) e^{\omega t}\right), \\
& v(\xi, y, t)=-v_{\mathrm{ff}} \cdot \tau_{0}(\xi)\left(1+\varepsilon \lambda_{\tau}(\xi) e^{\omega t}\right),
\end{aligned}
$$




$$
\begin{aligned}
P(\xi, y, t) & =\rho_{\mathrm{a}} v_{\mathrm{ff}}^{2} \cdot \pi_{0}(\xi)\left(1+\varepsilon \lambda_{\pi}(\xi) e^{\omega t}\right), \\
P_{\mathrm{e}}(\xi, y, t) & =\rho_{\mathrm{a}} v_{\mathrm{ff}}^{2} \cdot \pi_{\mathrm{e}}(\xi)\left(1+\varepsilon \lambda_{\mathrm{e}}(\xi) e^{\omega t}\right) .
\end{aligned}
$$

Thus we obtain the first order perturbed time-dependent equation

$$
\begin{aligned}
\frac{d}{d \tau_{0}}\left[\begin{array}{c}
\lambda_{\zeta} \\
\lambda_{\tau} \\
\lambda_{\pi} \\
\lambda_{\mathrm{e}}
\end{array}\right]= & \frac{1}{\tilde{\Lambda}}\left[\begin{array}{cccc}
1 & -1 & 0 & \frac{1}{\tau_{0}} \\
-\frac{\gamma \pi_{0}}{\tau_{0}} & 1 & -\frac{1}{\tau_{0}} & 0 \\
\gamma & -\gamma & \frac{1}{\pi_{0}} & 0 \\
\gamma & -\gamma & \frac{\gamma}{\tau_{0}} & -\frac{\left(\gamma \pi_{0}-\tau_{0}\right)}{\tau_{0} \pi_{\mathrm{e}}}
\end{array}\right] \\
& \times\left[\begin{array}{c}
F_{1} \\
F_{2} \\
F_{4} \\
F_{5}
\end{array}\right]
\end{aligned}
$$

(see Saxton $\&$ Wu 1999), where $\tilde{\Lambda} \equiv(\gamma-1) \Lambda\left(x_{\mathrm{s} 0} / \rho_{a} v_{\mathrm{ff}}^{3}\right)$ and $\tau_{0} \equiv-v_{0} / v_{\mathrm{ff}}$ are dimensionless versions of the cooling function and accretion flow velocity. The $F_{1}, \ldots, F_{5}$ terms are complex functions involving the perturbed variables and the variables of the stationary solution.

The differential equations are integrated from the shock down to the lower boundary. Values of $\delta$ which satisfy the appropriate boundary conditions are the eigenfrequencies. The complex eigenvalues $\delta=\delta_{\mathrm{R}}+i \delta_{\mathrm{I}}$ describe the oscillatory properties of the shock in different modes. The real part, $\delta_{R}$, is a growth term: if $\delta_{R}>0$ then the shock is unstable to oscillations in the corresponding mode. The imaginary part, $\delta_{\mathrm{I}}$, is proportional to the oscillatory frequency of the mode. Figure 1 shows examples of the eigenplanes of the perturbed variables $-\log \left|\lambda_{\tau}\right|$ evaluated for a given set of boundary conditions. The $\lambda$-profiles corresponding to the $\delta$ eigenvalues are the eigenfunctions of the post-shock structure's local oscillatory response to the shock oscillations.

For each cooling process, the luminosity response is calculated by multiplying the stationary-state cooling function and corresponding perturbation, and then integrating their product over the entire post-shock region. The luminosity response of a mode is expressed in terms of the stationary-solution luminosity and normalised by the size of the shock-height oscillation, i.e.

$$
\begin{aligned}
\frac{L_{\mathrm{br}, 1}}{\varepsilon L_{\mathrm{br}, 0}} & =\frac{\int_{0}^{1} \Lambda_{\mathrm{br}} \lambda_{\mathrm{br}} d \xi}{\int_{0}^{1} \Lambda_{\mathrm{br}} d \xi}, \\
\frac{L_{\mathrm{cy}, 1}}{\varepsilon L_{\mathrm{cy}, 0}} & =\frac{\int_{0}^{1} \Lambda_{\mathrm{cy}} \lambda_{\mathrm{cy}} d \xi}{\int_{0}^{1} \Lambda_{\mathrm{cy}} d \xi}
\end{aligned}
$$

where $\lambda_{\text {br }}=\frac{3}{2} \lambda_{\zeta}+\frac{1}{2} \lambda_{\mathrm{e}}$ and $\lambda_{\text {cy }}=-2.35 \lambda_{\zeta}+2.5 \lambda_{\mathrm{e}}$ (see Saxton \& Wu 2001). The absolute value of the ratio (14) is the relative amplitude of the bremsstrahlung (cyclotron) luminosity oscillation, and the phase $\Phi_{\mathrm{br}}$ (or $\Phi_{\mathrm{cy}}$ ) is relative to the oscillatory phase of the shock motion.

\section{Boundary Conditions}

\subsection{Shock Jump Condition}

The boundary conditions at the shock $(\xi=1)$ are determined by the strong-shock Rankine-Hugoniot conditions. It can be shown that the stationary solution conditions are $\tau_{0}=\frac{1}{4}, \pi_{0}=\frac{3}{4}$. If the ratio of electron and ion pressures is parameterised as $\sigma_{\mathrm{s}}$ then the electron pressure condition is $\pi_{\mathrm{e}}=\frac{3}{4} /\left(1+\sigma_{\mathrm{s}}^{-1}\right)$. The conditions on the perturbed variables are derived in the frame that is comoving with the shock (Imamura et al. 1996; see also appendices of Saxton, Wu, \& Pongracic 1997), $\lambda_{\zeta}=0, \lambda_{\tau}=-3$, and $\lambda_{\pi}=\lambda_{\mathrm{e}}=2$. The system parameters are the same as defined in Saxton \& Wu (1999): $\epsilon_{\mathrm{s}}$ is the efficiency of cyclotron cooling relative to bremsstrahlung cooling at the shock; $\sigma_{\mathrm{s}}$ is the ratio of electron to ion pressures at the shock; $\psi_{\text {ei }}$ specifies the efficiency of electron-ion energy exchange relative to the total efficiency of radiative cooling. The shock boundary conditions are the same in all cases studied; alternative conditions at the lower boundary are considered.

\subsection{Lower Boundary Conditions}

The post-shock flow has a lower boundary, labelled $\xi=0$, and the form and value of the boundary conditions there depend on the nature of the system under consideration. For example, the boundary of cooled gas far downstream from an interstellar medium shock and the boundary formed by a stellar surface in an accretion flow may impose different conditions on the hydrodynamic variables.

\subsection{1 'Perfect Stationary Wall'}

If the flow velocity vanishes at the lower boundary then the condition is $\tau_{0}=0$ at $\xi=0$. This is sometimes known as the 'stationary wall' condition. The additional choice of $\lambda_{\tau}=0$ means that the flow velocity is absolutely zero and does not oscillate at the lower boundary (case 1, Table 1). The 'perfect stationary wall' boundary condition is the conventional choice in many stability analyses of radiative shocks (e.g. Chevalier \& Imamura 1982; Tóth \& Draine 1993; Imamura et al. 1996; Saxton et al. 1998). Bertschinger (1986) demonstrated that this condition also applies to an ISM radiative shock at the inner boundary which is defined as the place where gas is cooled to a point where total cooling is zero.

Wolff, Gardner, \& Wood (1989) carried out numerical studies of accretion onto white dwarfs where cyclotron cooling does not dominate, and bremsstrahlung cooling, Compton cooling, electron-ion energy exchanges, and electron thermal conduction are included. They considered a perfect stationary wall condition at the lower boundary, with additional constraints: (i) a constant nonzero electron temperature $T_{\mathrm{e}}=T_{\text {cut }}$, the cutoff value where the total cooling $\Lambda=0$; (ii) a zero gradient in temperature, $\partial T_{\mathrm{e}} / \partial r=0$. The presence of conduction and the alternative 

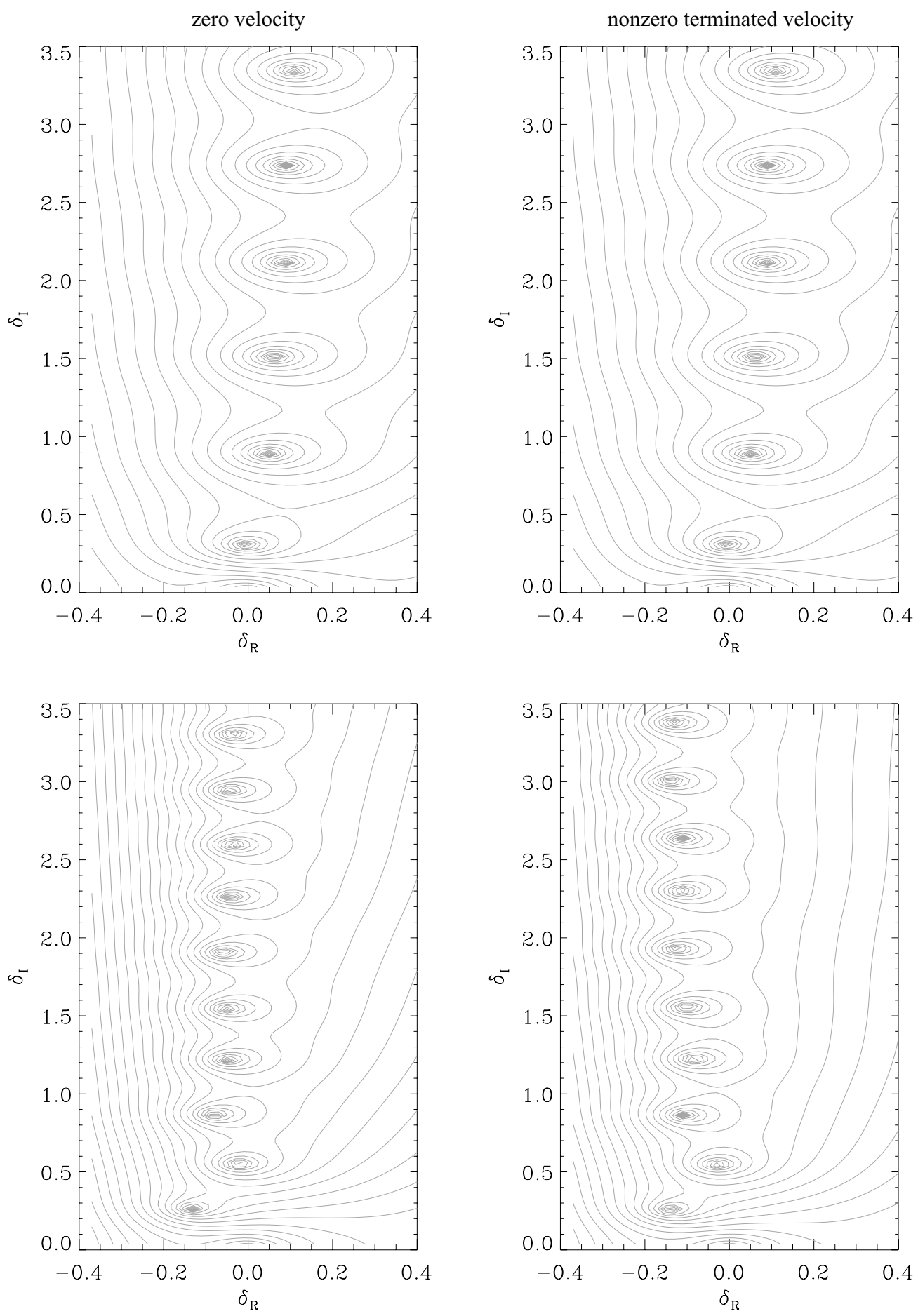

Figure 1 Complex frequency planes showing the eigenmodes of systems with parameters $\left(\sigma_{\mathrm{s}}, \psi_{\mathrm{ei}}, \epsilon_{\mathrm{s}}\right)=(0.5,0.5,0)$ in the top row and $\left(\sigma_{\mathrm{s}}, \psi_{\mathrm{ei}}, \epsilon_{\mathrm{s}}\right)=(0.5,0.5,100)$ in the bottom row. The lower boundary condition is $\lambda_{\tau}=0, \tau_{0}=0$ in the left column, and $\lambda_{\tau}=0, \tau_{0}=0.01$ in the right column. The contour levels are at logarithmic intervals of the function $1 /\left|\lambda_{\tau}\right|$; the modes are at the peaks. The real part of the eigenvalue, $\delta_{\mathrm{R}}$, describes the instability of a mode: positive indicates instability. The imaginary part of the eigenvalue, $\delta_{\mathrm{I}}$, is proportional to the frequency of oscillation.

form of $\Lambda$ must provide a solution for time-independent flow structure that is significantly different from that considered in the present work, which focuses on the role of lower boundary conditions on the perturbed variables. The effects of boundary conditions on stability properties of structures where conduction and finite cooling cutoffs are physically important comprises a significant topic for future investigation.

\subsubsection{Modified Stationary Wall}

The $\lambda_{\tau}=0$ fixed velocity assumption is not the only choice of lower boundary condition which is consistent with the 
Table 1. The boundary conditions studied in this paper, in terms of the stationary and perturbed variables

\begin{tabular}{|c|c|c|c|c|}
\hline \multirow{2}{*}{$\frac{\text { Case }}{1}$} & \multicolumn{2}{|c|}{ Stationary variables } & \multirow{2}{*}{$\begin{array}{c}\text { Perturbed variables } \\
\lambda_{\tau}=0\end{array}$} & \multirow{2}{*}{$\frac{\text { Note }}{\S 3.2 .1 \text { 'perfect stationary wall' }}$} \\
\hline & $\tau_{0}=0$, & $\pi_{0}=1$ & & \\
\hline 2 & $\tau_{0}=0$ & $\pi_{0}=1$ & $\lambda_{\pi}=0$ & $\$ 3.2 .2$ fixed pressure \\
\hline 3 & $\tau_{0}=0$ & $\pi_{0}=1$ & $\lambda_{\zeta}+\lambda_{\tau}=0$ & $\S 3.2 .2$ fixed flow rate \\
\hline 4 & $\tau_{0}=0$ & $\pi_{0}=1$ & $\lambda_{\zeta}=0$ & $\S 3.2 .2$ fixed density \\
\hline 5 & $\tau_{0}=0$ & $\pi_{0}=1$ & $\lambda_{\pi}-\lambda_{\zeta}=0$ & $\S 3.2 .2$ fixed temperature \\
\hline 6 & $\tau_{0}=0.01$ & $\pi_{0}=0.99$ & $\lambda_{\tau}^{3}=0$ & $\begin{array}{l}\$ 3.2 .3 \text { nonzero terminated } \\
\text { velocity; 'leaky flow' }\end{array}$ \\
\hline
\end{tabular}

stationary solution that provides $\tau_{0}=0$ at $\xi=0$. There are alternative physical propositions.

The stationary solution pressure at the lower boundary is a well-defined value, $\pi_{0}=1$. The condition $\lambda_{\pi}=0$ means that the total pressure of the flow does not oscillate at the lower boundary (case 2, Table 1). This is a kind of hydrostatic condition. In the case of accreting white dwarfs, this condition would mean that the pressure wave of the shock oscillation does not enter the white dwarf atmosphere.

The condition $\lambda_{\zeta}=0$ means that the density does not oscillate at the lower boundary (case 4 , Table 1). The condition $\lambda_{\zeta}+\lambda_{\tau}=0$ stands for constancy of the mass flow rate $\rho v$ at the lower boundary (case 3, Table 1). The physical interpretation of these two choices has a subtle complication: in the present treatment the density grows indefinitely as $\xi \rightarrow 0$. However this problem would be alleviated in a modified geometry (e.g. taking account of lateral flow from the sides of the accretion column) which will be investigated in future studies. (The present model applies to white dwarf accretion shocks so long as lateral spreading of accreted gas is much slower than infall at the shock, and buildup of material at the base is slow on the cooling and oscillatory timescales.)

Other physical lower boundary conditions are related to the thermal structure of the post-shock flow. The electron and ion temperatures must be equal at the white dwarf surface, which implies that $\lambda_{\mathrm{e}}-\lambda_{\pi}=0$ is a necessary boundary condition. However this condition is satisfied for every choice of $\delta$, since the functional form of the electron-ion energy exchange $\Gamma$ ultimately exceeds the bremsstrahlung cooling $\Lambda_{\text {br }}$ in the high density, low temperature region near the lower boundary (see e.g. Saxton $\& \mathrm{Wu} 2001$ ). Another choice would be to fix the flow temperature at the lower boundary, assuming that the gas at the base of the flow is in thermal equilibrium with the white dwarf, and that the white dwarf surface is a perfect heat-bath/heat-sink. Constancy of $T \propto P / \rho$ implies a condition of $\lambda_{\pi}-\lambda_{\zeta}=0$ (case 5, Table 1). The lower boundary temperature is zero in the stationary solution for the present example, so this choice of condition on the perturbed variables describes the lower boundary as being a perfect node in the oscillation of the temperature profile.

\subsubsection{Nonzero Terminated Velocity}

This paper also considers an example in which the lower boundary has a nonzero flow velocity relative to the shock. This condition describes a system where cooling at the base does not compress the gas indefinitely, and the accretion shock moves outwards from the white dwarf surface (until non-linear effects become important or the shock becomes indistinct). Alternatively the boundary condition could describe a surface at which post-shock material is removed from the system at a nonzero rate, like flow into a 'leaky' bucket. The illustrative choice considered here is $\tau_{0} \rightarrow 0.01$ rather than zero, and $\lambda_{\tau}=0$ at the lower boundary (case 6 , Table 1 ). In other words, the shock propagates away from the white dwarf surface at a velocity that is 0.01 times the free-fall velocity of pre-shock matter, or else gas is removed from the accretion stream where it contacts the stellar surface at incident velocity $0.01 v_{\mathrm{ff}}$.

\section{Stability Properties}

\subsection{Eigenfunctions in the Standard Case}

In the perfect stationary wall case, there exist amplitude nodes and antinodes in the eigenfunctions for perturbed density $\left(\left|\lambda_{\zeta}\right|\right)$, and also for electron pressure $\left(\left|\lambda_{e}\right|\right)$ in cases when the two-temperature effects are important. The number of nodes depends on the harmonic number of the mode. Phases of the eigenfunctions change abruptly at the node locations, and wind gradually in intervening regions.

Apart from the perturbed velocity $\left(\lambda_{\tau}=0\right.$ by the boundary condition) the perturbed variables are not explicitly determined at the lower boundary, but have finite amplitudes within an order of magnitude of their typical amplitudes throughout the whole post-shock region. The perturbed electron pressure and total pressure variables always meet at the same value at the lower boundary, regardless of the boundary condition, because the electron and ion temperatures equilibrate at the lower boundary. For comprehensive discussion of the eigenfunctions, see Saxton (1999) and Saxton \& Wu (2001).

\subsection{Sensitivity of Eigenfunctions}

Saxton (1999, Sections 5.4.1-5.4.2) considered the relationships between eigenvalues and local features of the corresponding eigenfunctions. The relevant findings were as follows. 
For arbitrary alterations of the lower boundary condition resulting in a small offset of the eigenvalue, $|\Delta \delta|=$ 0.01 , the eigenfunctions alter significantly in the region $\xi \lesssim 0.05$ near the lower boundary, but remain essentially unchanged throughout the majority of the post-shock region $(0.05 \lesssim \xi \leq 1)$. In the sensitive base region, offsets from the perfect stationary wall condition yield steeper gradients in the amplitudes and phases of all the $\lambda$ variables. The amplitudes $\left|\lambda_{\zeta}\right|$ and $\left|\lambda_{\tau}\right|$ may differ in their ordinary values by over an order of magnitude; the pressure amplitudes $\left|\lambda_{\pi}\right|$ and $\left|\lambda_{\mathrm{e}}\right|$ differ by factors of a few.

At more extremely different boundary conditions, resulting in eigenvalues that are intermediate between modes of the perfect stationary wall case, the sensitive base region $(\xi \lesssim 0.03)$ with steep gradients in the $\lambda$ functions persists. Amplitudes are enhanced in regions up to $\xi \lesssim 0.85$, but this is not as significant as the efffects in the base region. The number of antinodes and nodes are comparable to those of the next higher modes of the perfect stationary wall case, but their positions are completely different.

Thus small offsets of the global frequency and stability properties alter the local behaviour of the lowest few per cent of the post-shock flow, but have no significant effects in regions of the flow further from the lower boundary.

\subsection{Eigenvalues: Stability and Frequencies}

The previous subsection reviewed how eigenfunctions of the hydrodynamic variables generally respond to alterations of lower boundary conditions resulting in small and large alterations of the frequencies and stability properties. This section details the effects on the eigenvalues, the mode frequencies, and stability properties, of specific alternatives for the lower boundary condition.

\subsubsection{Perfect Stationary Wall}

The general stability and frequency results for the standard perfect stationary wall lower boundary condition are as follows (see e.g. Chevalier \& Imamura 1982; Imamura et al. 1996; Saxton et al. 1997, 1998; Saxton \& Wu 1999, 2001). When bremsstrahlung emission is the only cooling process the fundamental mode is stable, and the overtones are unstable, with instability $\delta_{\mathrm{R}}$ tending to increase with harmonic number. When two-temperature effects are unimportant $\left(\psi_{\mathrm{ei}}>1\right)$, increasing the cyclotron cooling efficiency $\left(\epsilon_{\mathrm{s}}\right)$ stabilises each mode. Some modes stabilise more readily than others and the trend of $\delta_{\mathrm{R}}$ increasing with harmonic number breaks down more extensively as cyclotron cooling becomes more efficient. In the absence of two-temperature effects, the oscillatory frequencies are quantised like those of a pipe open at one end (Tóth \& Draine 1993; Saxton et al. 1997; Saxton \& Wu 1999). Increasing $\epsilon_{\mathrm{s}}$ reduces the frequency intervals between consecutive modes (Saxton et al. 1997; Saxton \& Wu 1999). When two-temperature effects are important $\left(\psi_{\mathrm{ei}} \lesssim 1\right)$, greater $\epsilon_{\mathrm{s}}$ may actually destabilise modes (Imamura et al. 1996) and the frequency sequence becomes more like that of a doubly-open or doubly-closed pipe (Saxton \& Wu 1999).

Small offsets of the $\delta$ values may produce large changes in the $\lambda$-profiles in the region near the lower boundary. The affected region is only a few per cent of the entire post-shock flow. For larger $\delta$ offsets the $\lambda$-profiles are qualitatively similar to the eigenfunctions of the nearest higher mode, except that: (1) the perturbed variables change abruptly near the lower boundary, as for small offsets; (2) throughout most of the post-shock region there is a general increase of amplitude with decreasing $\xi$; and (3) the nodes and antinodes are located at different positions. Thus systems with eigenfrequencies and stability properties very different from the stationary wall case can nonetheless have similar local oscillatory responses throughout most of the post-shock region except for a small base region.

\subsubsection{Modified Stationary Wall}

Table 2 presents eigenvalues for the alternative boundary conditions described in Section 3.2.2: constant pressure, flow rate, density, or temperature. Compared to the perfect stationary wall case, all of the modified stationary wall conditions yield complex $\delta$ values that differ by only a few per cent. The changes of $\delta$ are smaller than or comparable to the idealised 'small offset' cases (Section 4.2 above; also Saxton 1999, Section 5.4.1).

Thus the alteration of the lower boundary condition on perturbed variables has no significant effect on the global stability properties and oscillatory frequencies of the modes. The nature and efficiencies of the radiative cooling processes and the electron-ion energy exchange have a greater influence on the frequencies and stability properties of the oscillating shocks than the lower boundary conditions. Observed frequency ratios and damping characteristics of shock oscillations (e.g. in accretion onto magnetic white dwarfs) is expected to be practically independent of the lower boundary condition. (However the lower boundary may still have significant effects upon the modulation of bremsstrahlung and cyclotron luminosities, as discussed in Section 5.)

\subsubsection{Nonzero Terminated Velocity}

If the flow velocity terminates with a nonzero value, $\tau_{0}=0.01$, at the lower boundary (Section 3.2.3), the oscillatory frequencies are essentially unchanged, and the stability properties change slightly. For the cases studied, when bremsstrahlung emission is the only cooling process, the difference between zero-velocity termination and nonzero-velocity termination is a few times 0.001 in both $\delta_{\mathrm{R}}$ and $\delta_{\mathrm{I}}$; for $\epsilon_{\mathrm{S}}=100$ the changes are a few times 0.01 . Changing from zero-velocity termination to nonzero-velocity termination at the lower boundary made every mode more stable. Higher order modes are more significantly affected, and the effects are greatest when $\epsilon_{\mathrm{S}}$ is higher. As seen in Figure 1, the sequence of the modes' stability properties is qualitatively different for the zero- and nonzero-terminated velocity 
Table 2. Eigenvalues for four alternative choices of the lower boundary condition in the perturbed variables, but with the standard conditions on the stationary solution $\left(\tau_{0}=0, \pi_{0}=1, \pi_{\mathrm{e}}=\frac{1}{2}\right.$ at $\left.\xi=0\right)$. Values shown are for the first six harmonics, $n=1, \ldots, 6$

\begin{tabular}{|c|c|c|c|c|c|c|c|c|c|c|}
\hline \multirow[t]{2}{*}{$\sigma_{\mathrm{s}}$} & \multirow[t]{2}{*}{$\psi_{\mathrm{ei}}$} & \multirow[t]{2}{*}{$\epsilon_{\mathrm{s}}$} & \multicolumn{2}{|c|}{$\lambda_{\pi}=0$} & \multicolumn{2}{|c|}{$\lambda_{\zeta}+\lambda_{\tau}=0$} & \multicolumn{2}{|c|}{$\lambda_{\zeta}=0$} & \multicolumn{2}{|c|}{$\lambda_{\pi}-\lambda_{\zeta}=0$} \\
\hline & & & $\delta_{\mathrm{R}}$ & $\delta_{\mathrm{I}}$ & $\delta_{\mathrm{R}}$ & $\delta_{\mathrm{I}}$ & $\delta_{\mathrm{R}}$ & $\delta_{\mathrm{I}}$ & $\delta_{\mathrm{R}}$ & $\delta_{\mathrm{I}}$ \\
\hline \multirow[t]{6}{*}{0.5} & \multirow[t]{6}{*}{0.5} & \multirow[t]{6}{*}{0} & -0.006 & 0.305 & -0.007 & 0.307 & -0.006 & 0.306 & -0.006 & 0.306 \\
\hline & & & 0.048 & 0.890 & 0.047 & 0.895 & 0.048 & 0.892 & 0.048 & 0.892 \\
\hline & & & 0.063 & 1.507 & 0.060 & 1.514 & 0.061 & 1.511 & 0.061 & 1.511 \\
\hline & & & 0.087 & 2.111 & 0.084 & 2.121 & 0.086 & 2.117 & 0.086 & 2.117 \\
\hline & & & 0.091 & 2.728 & 0.086 & 2.741 & 0.088 & 2.736 & 0.088 & 2.736 \\
\hline & & & 0.109 & 3.334 & 0.104 & 3.351 & 0.107 & 3.345 & 0.107 & 3.345 \\
\hline \multirow[t]{6}{*}{0.5} & \multirow[t]{6}{*}{0.5} & \multirow[t]{6}{*}{1} & -0.064 & 0.331 & -0.066 & 0.333 & -0.065 & 0.332 & -0.065 & 0.332 \\
\hline & & & -0.018 & 0.820 & -0.020 & 0.825 & -0.019 & 0.823 & -0.019 & 0.823 \\
\hline & & & -0.013 & 1.385 & -0.017 & 1.393 & -0.016 & 1.389 & -0.016 & 1.389 \\
\hline & & & 0.013 & 1.921 & 0.009 & 1.935 & 0.011 & 1.930 & 0.011 & 1.930 \\
\hline & & & 0.011 & 2.481 & 0.003 & 2.496 & 0.006 & 2.490 & 0.006 & 2.490 \\
\hline & & & 0.035 & 3.030 & 0.028 & 3.054 & 0.031 & 3.047 & 0.031 & 3.047 \\
\hline \multirow[t]{6}{*}{0.5} & \multirow[t]{6}{*}{0.5} & \multirow[t]{6}{*}{100} & -0.128 & 0.269 & -0.137 & 0.269 & -0.135 & 0.264 & -0.135 & 0.264 \\
\hline & & & -0.023 & 0.526 & -0.024 & 0.554 & -0.022 & 0.551 & -0.022 & 0.551 \\
\hline & & & -0.070 & 0.859 & -0.085 & 0.876 & -0.081 & 0.868 & -0.081 & 0.868 \\
\hline & & & -0.045 & 1.164 & -0.053 & 1.226 & -0.049 & 1.218 & -0.049 & 1.218 \\
\hline & & & -0.072 & 1.505 & -0.056 & 1.556 & -0.051 & 1.547 & -0.051 & 1.547 \\
\hline & & & -0.063 & 1.859 & -0.065 & 1.919 & -0.059 & 1.907 & -0.059 & 1.907 \\
\hline
\end{tabular}

boundary conditions in the cyclotron-dominated extreme $\left(\sigma_{\mathrm{s}}, \psi_{\mathrm{ei}}, \epsilon_{\mathrm{s}}\right)=(0.5,0.5,100)$.

The increased stability of modes in the cases of cyclotron-dominated cooling may occur because the conditions at the lower boundary are not as dense as in the perfect stationary wall case. Since the highest densities near the lower boundary are less extreme, the bremsstrahlung cooling dominates over a smaller region at the base of the post-shock flow. As bremsstrahlung radiative cooling is responsible for the thermal instability that drives the shock oscillations, it is qualitatively sensible that the nonzero terminated velocity case has more stable modes than the perfect stationary wall case.

\section{Luminosity Response}

Oscillations of the post-shock flow structure modulate the emitted radiation. Rapid $\sim 1 \mathrm{~Hz}$ shock oscillations were observationally inferred in accretion shocks of white dwarfs in some AM Herculis (AM Her) systems by optical-infrared observations of the oscillating cyclotron luminosities (Middleditch 1982; Imamura \& SteimanCameron 1986; Larsson 1987, 1989; Ramseyer et al. 1993; Middleditch, Imamura, \& Steiman-Cameron 1997). However the shock oscillations of these systems may not produce comparable oscillations of bremsstrahlung luminosity. For some systems the X-ray luminosity responses are subject to upper limits of a few per cent (Wolff et al. 1999; Beardmore \& Osborne 1997). It is therefore interesting to predict the oscillatory responses of the bremsstrahlung and cyclotron total luminosities implied by the analytically obtained modes, and their phasing properties (Saxton \& Wu 2001).
Wolff et al. (1991) and Wood et al. (1992) carried out numerical investigations of noise-driven QPOs in cases where the magnetic field strength is low enough that the cyclotron radiative cooling does not dominate. They found that modes that are suppressed in linear analyses may be sustained by accretion noise and show significant luminosity responses. The luminosity responses were found to be linear, and bremsstrahlung and cyclotron amplitudes were comparable although bremsstrahlung signatures of the modes are broader and therefore less distinct in the frequency power spectrum.

The present work extends the previous analysis to consider how the luminosity oscillations may depend on the character of the lower boundary. The relative suppression of rapid oscillations in the X-rays may probe the nature of the boundary at the bottom of the post-shock accretion flow onto the white dwarf in AM Her systems.

The different radiative cooling processes and the electron-ion energy exchange are strong in different regions of the post-shock flow. Bremsstrahlung cooling depends on a high power of density, and therefore it peaks in the cold, high density region near the lower boundary. Cyclotron cooling depends on a high power of temperature, and therefore most of the cyclotron emission comes from the hot region near the shock. Therefore the local oscillations of hydrodynamic variables in the region near the lower boundary are effective at modulating the bremsstrahlung luminosity, whereas the oscillatory behaviour of the flow near the shock is most effective at modulating the cyclotron luminosity. Thus the bremsstrahlung luminosity and the cyclotron luminosity have different responses to the shock oscillation of any given mode. Furthermore, because different modes have 
different profiles of nodes, antinodes, and phases between the upper and lower boundaries, the oscillatory responses of the bremsstrahlung and cyclotron luminosities must differ between modes.

As shown in Saxton (1999), the oscillatory eigenfunctions are sensitive to changes of the lower boundary condition in the region close to the boundary $(\xi \lesssim 0.03)$ but insensitive in the rest of the post-shock flow $(0.03 \lesssim \xi \leq 1)$. Since bremsstrahlung emission peaks near the lower boundary and cyclotron emission peaks near the shock, it is to be expected that the integrated bremsstrahlung luminosity is sensitive to alterations of the lower boundary condition, but the cyclotron luminosity is not. The bremsstrahlung luminosity response is additionally sensitive to the lower boundary condition by way of the large amplitudes in the density eigenfunction $\lambda_{\zeta}$ that can occur at low $\xi$ for some $\delta$ frequency values. The calculated luminosity responses shown in Table 3 confirm this prediction when the perfect stationary wall condition $\left(\lambda_{\tau}=0\right)$ at the lower boundary is replaced

Table 3. Bremsstrahlung and cyclotron luminosity responses, for the first six modes, for various choices of the system parameters and lower boundary condition. For the blocks of values from left to right, top to bottom, the respective lower boundary conditions are $\lambda_{\pi}=0, \lambda_{\zeta}+\lambda_{\tau}=0, \lambda_{\zeta}=0, \lambda_{\pi}-\lambda_{\zeta}=0,\left(\lambda_{\tau}=0, \tau_{0}=0\right)$, and $\left(\lambda_{\tau}=0, \tau_{0}=0.01\right)$. The amplitudes are normalised by $\varepsilon$ and the phases are multiples of $\pi$ radians

\begin{tabular}{|c|c|c|c|c|c|c|c|c|c|c|}
\hline \multirow[t]{2}{*}{$\sigma_{\mathrm{s}}$} & \multirow[t]{2}{*}{$\psi_{\mathrm{ei}}$} & \multirow[t]{2}{*}{$\epsilon_{\mathrm{s}}$} & \multicolumn{4}{|c|}{$\lambda_{\pi}=0$} & \multicolumn{4}{|c|}{$\lambda_{\zeta}+\lambda_{\tau}=0$} \\
\hline & & & $\frac{\left|L_{\mathrm{br}, 1}\right|}{\varepsilon L_{\mathrm{br}, 0}}$ & $\Phi_{\mathrm{br}}$ & $\frac{\left|L_{\mathrm{cy}, 1}\right|}{\varepsilon L_{\mathrm{cy}, 0}}$ & $\Phi_{\mathrm{cy}}$ & $\frac{\left|L_{\mathrm{br}, 1}\right|}{\varepsilon L_{\mathrm{br}, 0}}$ & $\Phi_{\mathrm{br}}$ & $\frac{\left|L_{\mathrm{cy}, 1}\right|}{\varepsilon L_{\mathrm{cy}, 0}}$ & $\Phi_{\text {cy }}$ \\
\hline \multirow[t]{6}{*}{0.5} & \multirow[t]{6}{*}{0.5} & \multirow[t]{6}{*}{0} & 3.257 & 0.347 & - & - & 18.303 & -0.622 & - & - \\
\hline & & & 9.652 & -0.968 & - & - & 9.318 & -0.015 & - & - \\
\hline & & & 11.742 & -0.336 & - & - & 7.515 & 0.690 & - & - \\
\hline & & & 12.355 & 0.259 & - & - & 7.168 & -0.655 & - & - \\
\hline & & & 12.881 & 0.962 & - & - & 6.567 & -0.042 & - & - \\
\hline & & & 15.137 & -0.444 & - & - & 5.283 & 0.634 & - & - \\
\hline \multirow[t]{6}{*}{0.5} & \multirow[t]{6}{*}{0.5} & \multirow[t]{6}{*}{1} & 7.391 & 0.485 & 4.075 & -0.993 & 15.852 & -0.744 & 4.061 & -0.996 \\
\hline & & & 8.167 & 0.859 & 0.521 & 0.825 & 7.924 & -0.017 & 0.506 & 0.823 \\
\hline & & & 9.930 & -0.434 & 0.185 & 0.074 & 7.384 & 0.564 & 0.194 & 0.077 \\
\hline & & & 11.913 & 0.062 & 0.344 & -0.024 & 5.919 & -0.808 & 0.343 & -0.023 \\
\hline & & & 10.855 & 0.718 & 0.339 & -0.052 & 6.044 & -0.218 & 0.339 & -0.055 \\
\hline & & & 14.405 & -0.727 & 0.329 & -0.104 & 4.845 & 0.374 & 0.331 & -0.105 \\
\hline \multirow[t]{6}{*}{0.5} & \multirow[t]{6}{*}{0.5} & \multirow[t]{6}{*}{100} & 24.160 & 0.461 & 3.441 & 0.987 & 17.381 & 0.934 & 3.304 & 0.967 \\
\hline & & & 18.551 & 0.485 & 0.474 & -0.657 & 2.268 & -0.357 & 0.420 & -0.578 \\
\hline & & & 7.654 & -0.752 & 0.930 & -0.208 & 4.668 & 0.156 & 0.966 & -0.202 \\
\hline & & & 19.076 & -0.519 & 1.164 & -0.231 & 3.054 & 0.537 & 1.174 & -0.229 \\
\hline & & & 15.504 & -0.224 & 1.146 & -0.216 & 3.195 & -0.943 & 1.139 & -0.211 \\
\hline & & & 13.482 & 0.292 & 1.088 & -0.191 & 3.602 & -0.568 & 1.077 & -0.189 \\
\hline \multirow[t]{2}{*}{$\sigma_{\mathrm{s}}$} & \multirow[t]{2}{*}{$\psi_{\mathrm{ei}}$} & \multirow[t]{2}{*}{$\epsilon_{\mathrm{s}}$} & \multicolumn{4}{|c|}{$\lambda_{\zeta}=0$} & \multicolumn{4}{|c|}{$\lambda_{\pi}-\lambda_{\zeta}=0$} \\
\hline & & & $\frac{\left|L_{\mathrm{br}, 1}\right|}{\varepsilon L_{\mathrm{br}, 0}}$ & $\Phi_{\mathrm{br}}$ & $\frac{\left|L_{\mathrm{cy}, 1}\right|}{\varepsilon L_{\mathrm{cy}, 0}}$ & $\Phi_{\mathrm{cy}}$ & $\frac{\left|L_{\mathrm{br}, 1}\right|}{\varepsilon L_{\mathrm{br}, 0}}$ & $\Phi_{\mathrm{br}}$ & $\frac{\left|L_{\mathrm{cy}, 1}\right|}{\varepsilon L_{\mathrm{cy}, 0}}$ & $\Phi_{\text {cy }}$ \\
\hline \multirow[t]{6}{*}{0.5} & \multirow[t]{6}{*}{0.5} & \multirow[t]{6}{*}{0} & 2.721 & -0.375 & - & - & 2.729 & -0.376 & - & - \\
\hline & & & 1.657 & -0.757 & - & - & 1.647 & -0.755 & - & - \\
\hline & & & 2.468 & -0.446 & - & - & 2.452 & -0.477 & - & - \\
\hline & & & 1.247 & 0.134 & - & - & 1.231 & 0.132 & - & - \\
\hline & & & 1.631 & 0.938 & - & - & 1.613 & 0.937 & - & - \\
\hline & & & 1.946 & -0.466 & - & - & 1.925 & -0.467 & - & - \\
\hline \multirow[t]{6}{*}{0.5} & \multirow[t]{6}{*}{0.5} & 1 & 1.272 & -0.355 & 4.079 & -0.995 & 1.284 & -0.356 & 4.079 & -0.995 \\
\hline & & & 0.286 & 0.644 & 0.190 & 0.0077 & 0.277 & 0.635 & 0.513 & 0.825 \\
\hline & & & 1.103 & -0.807 & 0.513 & -0.825 & 1.099 & -0.811 & 0.190 & 0.077 \\
\hline & & & 0.906 & -0.226 & 0.344 & -0.024 & 0.897 & -0.232 & 0.344 & -0.024 \\
\hline & & & 1.093 & 0.429 & 0.339 & -0.054 & 1.085 & 0.425 & 0.339 & -0.054 \\
\hline & & & 0.984 & -0.944 & 0.331 & -0.105 & 0.969 & -0.949 & 0.331 & -0.105 \\
\hline 0.5 & 0.5 & 100 & 0.885 & -0.415 & 3.597 & 0.976 & 0.923 & -0.419 & 3.598 & 0.976 \\
\hline & & & 0.124 & -0.172 & 0.429 & -0.585 & 0.141 & -0.223 & 0.429 & -0.585 \\
\hline & & & 0.441 & 0.787 & 0.951 & -0.203 & 0.443 & 0.778 & 0.951 & -0.203 \\
\hline & & & 0.397 & -0.887 & 1.173 & -0.229 & 0.393 & -0.911 & 1.173 & -0.229 \\
\hline & & & 0.475 & -0.351 & 1.143 & -0.211 & 0.457 & -0.362 & 1.143 & -0.211 \\
\hline & & & 0.643 & 0.033 & 1.080 & -0.189 & 0.636 & 0.023 & 1.080 & $\begin{array}{r}-0.189 \\
(\text { Continued })\end{array}$ \\
\hline
\end{tabular}


Table 3. (Continued)

\begin{tabular}{|c|c|c|c|c|c|c|c|c|c|c|}
\hline \multirow[t]{2}{*}{$\sigma_{\mathrm{s}}$} & \multirow[t]{2}{*}{$\psi_{\mathrm{ei}}$} & \multirow[t]{2}{*}{$\epsilon_{\mathrm{s}}$} & \multicolumn{4}{|c|}{$\lambda_{\tau}=0$} & \multicolumn{4}{|c|}{$\lambda_{\tau}=0, \tau_{0}=0.01$} \\
\hline & & & $\frac{\left|L_{\mathrm{br}, 1}\right|}{\varepsilon L_{\mathrm{br}, 0}}$ & $\Phi_{\mathrm{br}}$ & $\frac{\left|L_{\mathrm{cy}, 1}\right|}{\varepsilon L_{\mathrm{cy}, 0}}$ & $\Phi_{\text {cy }}$ & $\frac{\left|L_{\mathrm{br}, 1}\right|}{\varepsilon L_{\mathrm{br}, 0}}$ & $\Phi_{\mathrm{br}}$ & $\frac{\left|L_{\mathrm{cy}, 1}\right|}{\varepsilon L_{\mathrm{cy}, 0}}$ & $\Phi_{\mathrm{cy}}$ \\
\hline \multirow[t]{6}{*}{0.5} & 0.5 & 0 & 2.745 & -0.373 & - & - & 2.718 & -0.363 & - & - \\
\hline & & & 1.618 & -0.766 & - & - & 1.723 & -0.782 & - & - \\
\hline & & & 2.497 & -0.458 & - & - & 2.664 & -0.454 & - & - \\
\hline & & & 1.306 & 0.112 & - & - & 1.451 & 0.122 & - & - \\
\hline & & & 1.641 & 0.911 & - & - & 1.797 & 0.909 & - & - \\
\hline & & & 1.983 & -0.490 & - & - & 2.139 & -0.490 & - & - \\
\hline \multirow{6}{*}{0.5} & 0.5 & 1 & 1.318 & -0.349 & 4.079 & -0.995 & 1.196 & -0.324 & 4.085 & -0.995 \\
\hline & & & 0.345 & 0.615 & 0.513 & 0.825 & 1.193 & -0.806 & 0.190 & 0.082 \\
\hline & & & 1.197 & -0.820 & 0.190 & 0.077 & 0.414 & 0.641 & 0.514 & 0.823 \\
\hline & & & 1.015 & -0.240 & 0.344 & -0.024 & 1.020 & -0.215 & 0.343 & -0.024 \\
\hline & & & 1.228 & 0.406 & 0.339 & -0.054 & 1.238 & 0.424 & 0.341 & -0.054 \\
\hline & & & 1.109 & -0.974 & 0.331 & -0.105 & 1.115 & -0.958 & 0.331 & -0.104 \\
\hline \multirow[t]{6}{*}{0.5} & 0.5 & 100 & 1.254 & -0.415 & 3.600 & 0.976 & 0.445 & -0.364 & 3.768 & 0.971 \\
\hline & & & 0.183 & -0.047 & 0.429 & -0.585 & 0.047 & -0.098 & 0.411 & -0.595 \\
\hline & & & 0.715 & 0.743 & 0.950 & -0.202 & 0.211 & 0.844 & 0.966 & -0.188 \\
\hline & & & 0.652 & -0.923 & 1.173 & -0.229 & 0.170 & -0.821 & 1.169 & -0.229 \\
\hline & & & 0.804 & -0.399 & 1.143 & -0.211 & 0.202 & -0.288 & 1.124 & -0.215 \\
\hline & & & 1.102 & -0.021 & 1.080 & -0.189 & 0.257 & 0.081 & 1.069 & -0.193 \\
\hline
\end{tabular}

by the alternative conditions described in Sections 3.2.2 and 3.2.3. The bremsstrahlung luminosity amplitudes and phasings change significantly under alterations of the lower boundary condition, but the cyclotron luminosity response is essentially unaffected.

Because of the insensitivity of cyclotron luminosity responses to the lower boundary condition, the following discussion concentrates on the bremsstrahlung response alone.

\subsection{Perfect Stationary Wall}

As discussed for the choice of a perfect stationary wall lower boundary in Saxton \& Wu (1999), the modes' luminosity responses were found to be insensitive to $\left(\sigma_{\mathrm{s}}, \psi_{\mathrm{ei}}\right)$ when bremsstrahlung cooling dominates $\left(\epsilon_{\mathrm{s}}=0\right)$. However there are no obvious relationships between the luminosity responses and stability properties of the modes for diverse cases of the system parameters. It was found that the cyclotron luminosity oscillation tends to lag the bremsstrahlung luminosity oscillation by approximately $0.6 \pi$ radians for the fundamental mode, but the overtones lack obvious relationships between their bremsstrahlung and cyclotron responses.

\subsection{Fixed Density or Fixed Temperature}

The bremssttrahlung luminosity responses are identical to within a few per cent for the cases of fixed density $\left(\lambda_{\zeta}=0\right)$ and fixed temperature $\left(\lambda_{\pi}-\lambda_{\zeta}=0\right)$ boundary conditions. Thus in this sense an absence of oscillation in density at the lower boundary is almost equivalent to an absence of oscillation in temperature. For each choice of $\left(\sigma_{\mathrm{s}}, \psi_{\mathrm{ei}}, \epsilon_{\mathrm{s}}\right)$ there is an approximately constant ratio between the $\left|L_{\mathrm{br}, 1}\right| / \varepsilon L_{\mathrm{br}, 0}$ values in the fixed density and

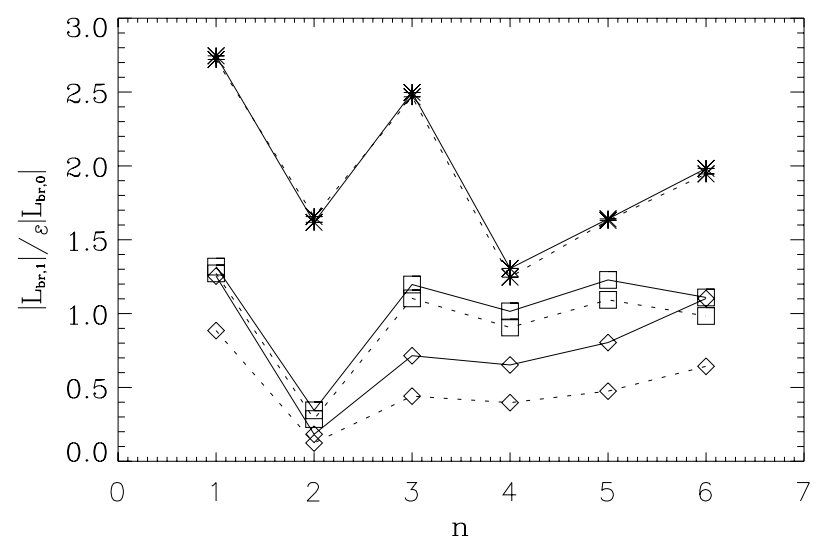

Figure 2 Comparison of bremsstrahlung luminosity oscillatory amplitude for cases with perfect stationary wall $\left(\lambda_{\tau}=0\right.$, solid lines) and fixed density ( $\lambda_{\zeta}=0$, dotted lines) lower boundary conditions. Values for the first six harmonics are marked with stars, squares, and diamonds for $\epsilon_{\mathrm{s}}=0,1,100$ respectively. The other system parameters are $\left(\sigma_{\mathrm{s}}, \psi_{\mathrm{ei}}\right)=(0.5,0.5)$. For given system parameters, the $\left|L_{\mathrm{br}, 1}\right| / \varepsilon L_{\mathrm{br}, 0}$ values in the fixed density case are approximately proportional to corresponding values in the perfect stationary wall case.

fixed temperature cases as compared to corresponding modes in the perfect stationary wall case (see Figure 2). For $\left(\sigma_{\mathrm{s}}, \psi_{\mathrm{ei}}, \epsilon_{\mathrm{s}}\right)=(0.5,0.5)$ the perfect stationary wall and fixed density/temperature $\left|L_{\mathrm{br}, 1}\right|$ values are in the approximate ratio of 1.0 for $\epsilon_{\mathrm{s}}=0,1.0-1.2$ for $\epsilon_{\mathrm{s}}=1$, and $1.3-1.7$ for $\epsilon_{\mathrm{s}}=100$. The phases of the bremsstrahlung luminosity responses are approximately equal for each mode in the fixed density/fixed temperature cases and the perfect stationary wall case. For the studied modes where $\left(\sigma_{\mathrm{s}}, \psi_{\mathrm{ei}}\right)=(0.5,0.5)$, the phase differences are $\lesssim 0.02 \pi$ radians for $\epsilon_{\mathrm{s}}=0, \lesssim 0.03 \pi$ radians for $\epsilon_{\mathrm{s}}=1$, and $\lesssim 0.04 \pi$ radians for $\epsilon_{\mathrm{s}}=100$. 


\subsection{Fixed Pressure}

With the present choices of system parameters, the bremsstrahlung luminosity of each mode is greater under the fixed pressure condition $\left(\lambda_{\pi}=0\right)$ than under the perfect stationary wall condition $\left(\lambda_{\tau}=0\right)$. Modes of higher harmonic number tend to have higher amplitudes of bremsstrahlung luminosity response. (One exception is the $n=3$ mode when $\left(\sigma_{\mathrm{s}}, \psi_{\mathrm{ei}}, \epsilon_{\mathrm{s}}\right)=(0.5,0.5,100)$, which is also unusual because the instability eigenvalue $\delta_{\mathrm{R}}$ departs from the usual trend of $\delta_{\mathrm{R}}$ increasing with harmonic number $n$.) The fixed pressure and perfect stationary wall lower boundary conditions also produce different bremsstrahlung luminosity phases. As shown in the topleft and bottom-left blocks of Table 3, the bremsstrahlung luminosity phases for the first six harmonics differ by $\sim 0.3 \pi-0.8 \pi$ for $\left(\sigma_{\mathrm{s}}, \psi_{\mathrm{ei}}\right)=(0.5,0.5)$ and $\epsilon_{\mathrm{s}}=1,100$. The change of boundary condition makes less difference ( $\lesssim 0.3 \pi$ in phase) for the same modes when cyclotron cooling is absent $\left(\epsilon_{\mathrm{s}}=0\right)$.

\subsection{Fixed Flow Rate}

In the cases with fixed flow rate boundary condition $\left(\lambda_{\zeta}+\lambda_{\tau}=0\right)$ the bremsstrahlung luminosity response amplitudes generally decrease with harmonic number, $n$. The exceptions, for the choices of system parameters studied here, are the modes which depart from the trend of $\delta_{\mathrm{R}}$ increasing with harmonic number, $n$. There is no clear relationship between the phases of bremsstrahlung luminosity oscillation in the perfect stationary wall and fixed flow cases, however the phase of each mode in the fixed flow case is approximately in antiphase to that in the corresponding fixed pressure case.

\subsection{Nonzero Terminated Velocity}

When cyclotron cooling is absent, $\left(\sigma_{\mathrm{s}}, \psi_{\mathrm{ei}}, \epsilon_{\mathrm{s}}\right)=(0.5$, $0.5,0)$, the bremsstrahlung luminosity responses for the nonzero terminated velocity condition $\left(\tau_{0}=0.01, \lambda_{\tau}=0\right)$ are almost the same as for the perfect stationary wall condition $\left(\tau_{0}=0, \lambda_{\tau}=0\right)$. The corresponding phases are approximately equal (to within $\lesssim 0.005 \pi$ radians, see Table 3 , bottom row). The amplitudes agree to within a few per cent.

When bremsstrahlung and cyclotron cooling are equally efficient at the shock $\left(\sigma_{\mathrm{s}}, \psi_{\mathrm{ei}}, \epsilon_{\mathrm{s}}\right)=(0.5,0.5,1)$, zero- and nonzero-velocity termination have different luminosity responses for the lowest three modes, but approximately equal luminosity responses for the higher order modes. When cyclotron cooling dominates, $\left(\sigma_{\mathrm{s}}, \psi_{\mathrm{ei}}, \epsilon_{\mathrm{s}}\right)=(0.5,0.5,100)$, the velocity termination affects the luminosity responses for all the modes studied.

It is not clear why nonzero velocity termination at the lower boundary affects luminosity responses of low harmonic modes more than higher modes. Higher modes have $\lambda_{\text {br }}$ profiles with more 'nodes' and 'antinodes', which should be expected to make them more sensitive to altered conditions at many locations in the post-shock flow; lower modes have fewer amplitude extrema and lesser spatial gradients of phase. It can be speculated that for modes of higher harmonic number, the integration in (14) involves a proportionately smaller contribution in the low $\xi$, compared to the case of lower modes.

\subsection{Comparison with Numerical Results}

In numerical studies of noise-driven shock oscillations in low field (low $\epsilon_{\mathrm{s}}$ ) cases, Wood et al. (1992) determined the lag of cyclotron luminosity response relative to the bremsstrahlung luminosity response, which is comparable to the difference $\Phi_{\text {cy }}-\Phi_{\text {br }}$ in the notation of the present paper. For cases with comparable equilibrium luminosities of cyclotron and bremsstrahlung $\left(L_{\mathrm{cy}, 0} / L_{\mathrm{br}, 0}=0.5\right)$ the cyclotron phase lag was $-0.8 \pi$ radians for the fundamental $(n=1)$ mode, and $-0.2 \pi$ for the first overtone $(n=2)$. This result is approximately consistent with the typical lag $\sim-0.6 \pi$ for the fundamental mode in the fixed density, fixed temperature, and fixed velocity lower boundary conditions for cases with $\epsilon_{\mathrm{s}}=1$ in Table 3, but inconsistent with fixed flow rate and fixed pressure conditions. Comparison of the cyclotron luminosity lags for the $n=2$ mode may not be meaningful, because in the analytic results the $n=2$ mode's phasing is sensitive to the system parameters as well as the lower boundary condition. Wood et al. (1992) also caution that phasing may differ between free and driven oscillations; by assumption, our analytically represented oscillations are physically free.

As Imamura (1985) recognised, the discrepant results from numerical investigations of radiative shock instabilities in accreting white dwarfs may be largely affected by the numerical treatment of the cold, high density gas near the lower boundary, where rapid cooling causes several of the fluid variables to experience sharp gradients. In the Lagrangian numerical hydrodynamic calculations of Imamura et al. (1984) the grid zones accumulated at the lower boundary were eliminated by coalescing the cells. In Imamura (1985) the cluttered grid zones were instead eliminated after cooling below a threshold temperature. For the latter method the shock stabilities were less sensitive to the procedural details than in the previous method. It is possible that methods involving mergers or eliminations of material accreted at the base of the flow have an effectively open lower boundary condition. If the merger/deletion process still conserves mass, energy, and momentum, then it still may effectively delete structural information such as the ideally steep gradients of velocity and density near the lower boundary. The importance of those gradients is shown by the different oscillatory responses of the luminosities, between the perfect stationary wall and nonzero terminated velocity boundary conditions.

The Eulerian calculations (e.g. Langer et al. 1981, 1982; Chanmugam, Langer, \& Shaviv 1985) necessarily introduced a spatial filter to remove noise and numerical instabilities. This process may also affect the thermal instability by smoothing away oscillatory structures, such as those in the sensitive region immediately above the lower boundary, that vary on finer spatial scales than the 
filtering resolution (e.g. nodes and phase jumps like those in the eigenfunctions of higher modes in Saxton \& Wu (2001)).

\section{Conclusions}

The oscillatory and stability properties are not significantly affected for modifications of the stationary wall boundary condition involving the perturbed hydrodynamic variables, for constant pressure, flow rate, density, or temperature at the lower boundary. The cases considered here have eigenvalues which are effectively identical to the perfect stationary wall results, or at most the 'small offset' profiles which only affect the $\lambda$-functions in regions very close to the lower boundary.

If the stationary solution terminates at a lower boundary with nonzero velocity, the oscillatory frequencies are essentially unchanged from the perfect stationary wall case. However the nonzero-terminated velocity modes are slightly more stable than the corresponding modes of the perfect stationary wall, and the higher modes are more greatly affected.

The cyclotron luminosity response is independent of the lower boundary condition as this emission is peaked near the shock, where jump conditions specify the hydrodynamic variables and the lower boundary conditions have little influence. The bremsstrahlung luminosity response is a sensitive probe of the conditions at the lower boundary, because bremsstrahlung emission is peaked near that boundary and the perturbed hydrodynamic variables in this region are locally very sensitive to changes in the lower boundary conditions. However the fixed density and fixed temperature lower boundary conditions have modes with identical luminosity responses; the reason for this equivalence is unclear.

Termination of the stationary solution at nonzero velocity produces effectively identical luminosity responses as the same modes with zero velocity termination, for all modes when cyclotron cooling is unimportant (i.e. when $\epsilon_{\mathrm{S}}$ is small). As $\epsilon_{\mathrm{s}}$ increases, the velocity termination makes a difference to the luminosity responses of the lowest order modes but not higher order modes. When $\epsilon_{\mathrm{s}}$ is sufficiently large, nonzero velocity termination affects the luminosity responses of all modes studied. For modest two-temperature effects, the bremsstrahlung luminosity responses of higher order modes tend (with few exceptions) to be greater under the fixed pressure lower boundary condition, and lower under the fixed flow rate lower boundary condition. However there is no universal pattern between $\delta$ and the luminosity responses for general $\left(\sigma_{\mathrm{s}}, \psi_{\mathrm{ei}}\right)$. For a given mode and given system parameters, the bremsstrahlung responses in the fixed pressure and fixed flow cases are approximately in antiphase.

Past numerical simulations reproduce some of what may be the important qualitative features of these thermally unstable radiative shocks, though it is not clear which, if any, of the numerical treatments provides superior predictions about the stability properties and frequencies of the shock oscillations. All the treatments to date may lack sufficient spatial and temporal resolution in the lower boundary region, where the oscillatory instability may originate. Indeed treatments may alter the fundamental character of the boundary (e.g. the removal of grid zones or accreted material may provide a more open boundary than would be realistic for the fixed wall of the white dwarf surface).

\section{Acknowledgments}

I thank Kinwah Wu, James Imamura, and the anonymous referee for thorough comments on the manuscript, which have contributed greatly to the paper's improvement and clarification.

\section{References}

Beardmore, A. P., \& Osborne, J. P. 1997, MNRAS, 286, 77

Bertschinger, E. 1986, ApJ, 304, 154

Chanmugam, G., Langer, S. H., \& Shaviv, G. 1985, ApJ, 299, L87

Chevalier, R. A., \& Imamura, J. N. 1982, ApJ, 261, 543

Cropper, M. 1990, SSRv, 54, 195

Field, G. B. 1965, ApJ, 142, 531

Imamura, J. N. 1985, ApJ, 296, 128

Imamura, J. N., \& Steiman-Cameron, T. Y. 1986, ApJ, 311, 786

Imamura, J. N., Wolff, M. T., \& Durisen, R. H. 1984, ApJ, 276,667

Imamura, J. N., Aboasha, A., Wolff, M. T., \& Wood, K. S. 1996, ApJ, 458,327

Langer, S. H., Chanmugam, G., \& Shaviv, G. 1981, ApJ, 245, L23

Langer, S. H., Chanmugam, G., \& Shaviv, G. 1982, ApJ, 258, 289

Larsson, S. 1987, A\&A, 181, L15

Larsson, S. 1989, A\&A, 217, 146

Melrose, D. B. 1986, Instabilities in Laboratory and Space Plasmas (Cambridge: Cambridge University Press)

Middleditch, J. 1982, ApJ, 257, L71

Middleditch, J., Imamura, J. N., \& Steiman-Cameron, T. Y. 1997, ApJ, 489, 912

Ramsayer, T. F., Robinson, E. L., Zhang, E., Wood, J. H., \& Stiening, R. F. 1993, MNRAS, 260, 209

Rybicki, G. B., \& Lightman, A. P. 1979, Radiative Processes in Astrophysics (New York: John Wiley \& Sons)

Saxton, C. J. 1999, PhD Thesis, University of Sydney

Saxton, C. J., \& Wu, K. 1999, MNRAS, 310, 677

Saxton, C. J., \& Wu, K. 2001, MNRAS, 324, 659

Saxton, C. J., Wu, K., \& Pongracic, H. 1997, PASA, 14, 164

Saxton, C. J., Wu, K., Pongracic, H., \& Shaviv, G. 1998, MNRAS, 299,862

Spitzer, L. 1962, Physics of Fully Ionized Gases (New York: Interscience), 135

Tóth, G., \& Draine, B. T. 1993, ApJ, 413, 176

Wolff, M. T., Gardner, J., \& Wood, K. S. 1989, ApJ, 346, 833

Wolff, M. T., Wood, K. S., \& Imamura, J. N. 1991, ApJ, 375, L31

Wolff, M. T., Imamura, J. N., Middleditch, J., Wood, K. S., \& Steiman-Cameron, T. 1999, Annapolis Workshop on Magnetic Cataclysmic Variables, ASP Conf. Series 157, eds C. Hellier, \& K. Mukai (San Francisco: ASP), 149

Wood, K. S., Imamura, J. N., \& Wolff, M. T. 1992, ApJ, 398, 593

Wu, K. 1994, PASA, 11, 61

Wu, K. 2000, SSRv, 93, 611 